Abstract

\title{
Pushing the Thermodynamics/Information Analogy: Entropy Behaviour of PDEs ${ }^{\dagger}$
}

\author{
Philip Broadbridge \\ La Trobe University, Melbourne, Australia \\ + Presented at the Entropy 2021: The Scientific Tool of the 21st Century, 5-7 May 2021; Available online: \\ https://sciforum.net/conference/Entropy2021/.
}

Published: 5 May 2021

It is easily seen that the heat-flow statement of 2 nd law of thermodynamics is equivalent to information irreversibility when $\mathrm{p}(\mathrm{x}, \mathrm{t})$ satisfies a general nonlinear 2 nd order diffusion equation. In many situations the viscosity solution of a nonlinear wave equation is that which maintains a specified conservation law and maximizes the entropy jump at a shock. There are some systems in which information is not monotonic and yet it would be helpful to view entropy as predominantly increasing: (i) Heat and mass transport in the plasma universe cannot be represented by classical diffusion with unbounded speed of propagation. The simplest phenomenological correction to the hyperbolic diffusion equation is predominantly irreversible. (ii) A pure quantum state satisfying the Schrödinger equation. By the Hopf-Cole transformation this is equivalent to the momentum equation of the irrotational Madelüng fluid. However interventionist measurement induces vorticity in the fluid, which dissipates along with entropy increase during collapse to an eigen-state. While vorticity is present a full description requires a vector potential as well as the Schrödinger potential. (iii) So-called fourth-order diffusion is information-irreversible only for an identified class of nonlinear diffusivities.

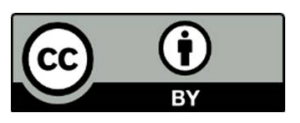

(C) 2021 by the author. Licensee MDPI, Basel, Switzerland. This article is an open access article distributed under the terms and conditions of the Creative Commons Attribution (CC BY) license (http://creativecommons.org/licenses/by/4.0/). 